\section{Livraisons}

d'Histoire

de l'Architecture

\section{Livraisons de l'histoire de l'architecture}

39 | 2020

Maquettes d'architecture

\title{
Les maquettes Perfecta, naissance d'un modèle
}

'Les maquettes Perfecta', birth of a model

Die "Perfecta"-Modelle, Entstehung eines Leitbildes

\section{Elvira Férault}

\section{OpenEdition}

\section{Journals}

Édition électronique

URL : http://journals.openedition.org//ha/1557

DOI : $10.4000 /$ lha. 1557

ISSN : 1960-5994

\section{Éditeur}

Association Livraisons d'histoire de l'architecture - LHA

Édition imprimée

Date de publication : 15 juin 2020

Pagination : 43-52

ISSN : $1627-4970$

\section{Référence électronique}

Elvira Férault, « Les maquettes Perfecta, naissance d'un modèle », Livraisons de l'histoire de l'architecture [En ligne], 39 | 2020, mis en ligne le 24 janvier 2021, consulté le 26 janvier 2021. URL: http://journals.openedition.org//ha/1557 ; DOl : https://doi.org/10.4000/lha.1557 


\section{LES MAQUETTES PERFECTA, NAISSANCE D’UN MODÈLE}

En 1935, le terme "maquettiste " ne figure pas encore dans le dictionnaire de l'Académie française. Il faut attendre l'année suivante pour qu'une première occurrence $^{1}$ fasse son apparition, signe des bouleversements en cours dans le milieu de la maquette d'architecture. La profession est alors tenue par des artisans - sculpteurs, mouleurs ou décorateurs - qui travaillent seuls et produisent des modèles généralement en plâtre. Un article intitulé "La maquette ", paru dans L'Architecture d'Aujourd'hui en 1932, n'hésite pas à affirmer : "Il existe en France un assez grand nombre d'artisans qui ne sont que des "maquettistes d'occasion", ainsi des modeleurs, des staffeurs, des mécaniciens, des décorateurs s'improvisent maquettistes, mais s'ils réussissent parfois, ils sont, en général, loin d'atteindre la perfection d'exécution à laquelle parviennent les hommes de métier $»^{2}$. Les lettres de candidature adressées au commissariat de l'Exposition internationale de $1937^{3}$ en témoignent également. Parmi les vingt-neuf courriers de demandes de travaux de modèles réduits, seuls six émanent d'entreprises spécialisées, parmi lesquels la société dénommée "Les Maquettes Perfecta».

L'étude de cet atelier, dont les origines remontent aux années vingt, conduit à interroger la notion d'innovation dans la pratique de la diffusion architecturale durant l'entre-deux-guerres. Extraordinaire vecteur de communication, l'utilisation de la maquette connaît en effet une véritable expansion sous l'impulsion des architectes du Mouvement moderne qui en font un usage central, relayé par l'emploi croissant de son image imprimée. L'enjeu ici est d'aborder l'histoire concrète de ces instruments - et de leur fabrication - qui donnèrent naissance à un corps de professionnels tel l'entreprise Perfecta, atelier emblématique de cette révolution. Si les sources ne sont pas nombreuses et si nous ne disposons malheureusement pas des archives privées de la société, nous tâcherons néanmoins d'en retracer l'aventure commerciale.

\section{Aux origines de la société}

À l'origine des maquettes Perfecta se trouve un homme, Joseph Sola. Né en février 1878 à Barcelone, il participe certainement à l'importante immigration espagnole vers l'hexagone au début du $\mathrm{XX}^{\mathrm{e}}$ siècle ${ }^{4}$. On retrouve sa trace à Paris au début

1. Alain Rey (dir.), Dictionnaire historique de la langue française, Paris, Le Robert, 2010.

2. Jean Léon, "Les maquettes", L'Architecture d'Aujourd'hui, n 2, mars 1932, p. 115.

3. Un sous-dossier "maquettistes" consigne les échanges entre l'administration de l'Exposition internationale de 1937 et des artisans en quête de travaux de maquettes (Arch. nat., F/12/12712).

4. À ce sujet : Marie-Catherine Talvikki Chanfreau, «Espagnols en territoire français de 1813 à 1971 : circuits ou intégrations d'exilés et d'émigrés ", Les Cahiers du MIMMOC, $\mathrm{n}^{\circ}$ 1, 2006. 
des années vingt alors qu'il multiplie les dépôts d'inventions auprès de l'Office national de la propriété industrielle. Parmi les six brevets ${ }^{5}$ de natures diverses qu'il conçoit coup sur coup, comme un "appareil moteur à charrues bascules parallèles " ou un "séchoir ménager pour linge ", Joseph Sola imagine en 1924 un "procédé pour fabrication de maquettes et modèles " qui permet de réaliser des maquettes d'architecture détaillées et réalistes, depuis le sol - terrain, clôture, relief et végétation - jusqu'à la toiture des bâtiments. Pour ce faire, il exclut toute utilisation du plâtre et prévoit au contraire l'emploi de matériaux courants et peu coûteux qui, grâce à un outillage réduit, ne nécessitent pas un "long travail de main-d'œuvre ". Il promet en outre la réalisation de modèles qui ne se brisent pas suite aux chocs, " ne se déforment pas sous l'effet des changements de température et d'humidité" et "sont d'un poids plus léger" que les maquettes alors produites. Il prévoit pour cela un socle en bois recouvert d'un épais carton dans lequel sont réalisées des entailles capables de recevoir des cloisons collées entre elles ou assemblées à l'aide d'agrafes. Afin d'en accentuer le réalisme, les façades sont peintes et peuvent être agrémentées de " petites bandes ou tiges carton ou bristol superposées ou collées » pour donner l'illusion des modénatures architecturales. Des feuilles de papier translucide sont également prévues à l'emplacement des ouvertures afin de « laisser voir par transparence l'éclairage électrique de l'intérieur des bâtiments " réalisé grâce à de petites ampoules introduites dans la base de la maquette. Cette invention, dont les caractéristiques semblent aujourd'hui élémentaires, est annonciatrice de la transformation formelle des maquettes au tournant des années trente. Généralement fabriquées en plâtre, elles s'apparentaient jusqu'alors davantage à des œuvres d'art et étaient d'ailleurs exposées comme telles, à l'image des maquettes blanches à la volumétrie orthogonale et aux finitions imparfaites présentées par Le Corbusier et Robert MalletStevens au Salon d'Automne de $1924^{6}$.

Quelques mois après le dépôt de son brevet, Sola se déclare auprès du greffe du tribunal de commerce ${ }^{7}$ comme artisan "modeleur pour fabriques " ${ }^{8}$. Installé à domicile, au 18 rue de Gergovie dans le $14^{\mathrm{e}}$ arrondissement de Paris, il se signale dès 1925 auprès de l'Annuaire du commerce Didot-Bottin comme ingénieur à la tête des Maquettes industrielles "Perfecta ", autrement dit "parfaites ", terme habilement choisi par son créateur ${ }^{9}$. L'annonce publiée dans l'almanach précise que les maquettes

5. En 1921, Joseph Sola dépose 3 brevets d'invention : "Appareil moteur à charrues bascules parallèles " ( ${ }^{\circ}$ 543.111, 2 mars 1921) ; "Brise-jet filtre " ( $n^{\circ}$ 534.671, 28 avril 1921) ; "Séchoir pour linge " ( $\mathrm{n}^{\circ}$ 536.562, 8 juin 1921). Il en dépose trois autres en $1924:$ "Filtre entonnoir à essence minérale " ( $n^{\circ}$ 576.127, 22 janvier 1924) ; "Procédé pour fabrication de maquettes et modèles " ( $n^{\circ} 582.239$, 14 avril 1924) et "Séchoir ménager pour linge" ( ${ }^{\circ} 582.262,21$ mai 1924), Archives INPI.

6. À ce sujet, Gérard Monnier, L'Architecture en France, une histoire critique 1918-1950, Paris, Sers, 1990, p. 259-262.

7. Joseph Sola régularise sa situation professionnelle en se déclarant auprès du tribunal de commerce en octobre 1924. À partir de la loi du 18 mars 1919 - complété par l'arrêté du 22 mars 1920 -, tout artisan ou commerçant était tenu de s'inscrire sur les registres du commerce et d'obtenir un numéro d'immatriculation. Il s'agissait à la fois d'un instrument de publicité mais également d'un moyen pour l'État de connaître de manière officielle l'existence des commerçants français ou étrangers.

8. Numéro d'immatriculation de Joseph Sola : A298713 (Arch. de Paris, D33U3/414).

9. On notera que le terme perfecta se comprend en français comme en espagnol et en catalan. 
sont "incassables ", réalisées à l'aide d'un procédé breveté sans plâtre qui permet de faire " $50 \%$ d'économie sur prix courants». À cette date, seuls cinq ateliers partagent la catégorie "plans en reliefs » de l'annuaire. Tous déclarent fournir les ministères, les musées, les facultés ainsi que les agences d'architecture. L'un d'eux ajoute qu'il a été médaillé à l'Exposition universelle de 1900, un autre qu'il est le successeur d'une maison déjà bien établie. Migrant, Joseph Sola n’hérite quant à lui ni d'une entreprise, ni d'un carnet de clients. Sans références, son annonce revêt un caractère strictement commercial.

Son affaire prend cependant de l'ampleur à tel point qu'il décide quelques années plus tard de s'associer à Jacques Pichat et Jean Léon, le neveu de Paul Léon, alors directeur général des Beaux-Arts, pour fonder la société anonyme "Les Maquettes Perfecta ", également dénommée "Anciens établissements Sola " ${ }^{10}$. Située 55 rue la Procession, à quelques pas de sa succursale qui n'est autre que son domicile, la société au capital de 200000 francs a pour objet « l'étude, la fabrication, l'achat et la vente de modèles en réduction, maquettes et tous articles de propagande et publicité ». Joseph Sola est toutefois rapidement écarté. Il ne figure plus dans les statuts de la société dès l'automne $1930^{11}$ et poursuit son activité de maquettiste dans le cadre de son entreprise individuelle, déclarée en faillite le 4 août $1932^{12}$. La société se désolidarise d'ailleurs immédiatement de son fondateur en diffusant une brève dans le quotidien Le Temps qui stipule : "La Société anonyme les "Maquettes Perfecta” (...) précise qu'elle n'a rien de commun avec M. Joseph Sola, anciennement constructeur des Maquettes Perfecta et déclaré en faillite ${ }^{13}$.

\section{Un atelier prospère}

Alors que les ateliers de maquettistes sont encore peu nombreux à Paris, Perfecta se développe de manière fulgurante. La société, qui profite de l'engouement des architectes pour des modèles de plus en plus réalistes, connaît une rapide et éclatante prospérité caractérisée par le doublement de son capital en un an avant d'atteindre un patrimoine de 350000 francs en $1935^{14}$. Florissant, l'atelier est déplacé et s'installe 53 bis rue de la Procession ${ }^{15}$ dans un immeuble flambant neuf. Ce changement d'adresse lui permet certainement d'agrandir ses espaces de travail. Il

10. La société anonyme est fondée en 1930 sous le numéro d'immatriculation B244847. Jacques Pichat sera associé durant trois années. Sa démission intervient le 23 décembre 1933 (Arch. de Paris, D33U3/1178).

11. La démission de Joseph Sola intervient le 19 septembre 1930 (Arch. de Paris, D33U3/1178).

12. L'immatriculation de Joseph Sola comme artisan est clôturée pour insuffisance d'actifs le 30 septembre 1932 (Arch. de Paris, D10U3/101).

13. Publication du 12 août 1932.

14. Le capital de la société est porté à 300000 francs en 1931 (Archives commerciales de la France, journal officiel d'annonces judicaires et légales, 25-27 mai 1931, p. 2571) puis à 350000 francs en 1935 (ibid., 30 octobre 1935, p. 1369).

15. Archives commerciales de la France, journal officiel d'annonces judicaires et légales, 21 mars 1934, p. 1484 . 
étoffe également ses stocks de matériaux et rachète " divers éléments d'un fonds de jouets et maquettes " lors de la vente d'un fonds de commerce ${ }^{16}$. Contraints par la rareté des sources, nous ne connaissons cependant pas le nombre d'employés, leurs profils et l'évolution des embauches. Un article publié dans L'Architecture d'Aujourd'hui ${ }^{17}$, dont l'auteur n'est autre que le dirigeant de la société, Jean Léon, permet pourtant de comprendre son organisation. Véritable opération d'autopromotion, le reportage, intitulé sobrement "Les maquettes ", décrit en réalité le fonctionnement de l'atelier. Jean Léon y détaille les tâches de chaque corps de métiers qui participent, en réduction, à un processus d'édification comparable à celui d'un projet architectural ${ }^{18}$. Il présente d'abord les dessinateurs qui "dissèquent " les documents fournis par les architectes afin de produire des plans de modèles réduits tout en "s'ingéniant à dissimuler par de savants artifices les parties les moins importantes" du projet. Il mentionne ensuite "les mécaniciens qui coupent, percent, tournent, façonnent, soudent le métal " puis "les menuisiers, les cartonniers, les électriciens qui préparent les éclairages ou les mouvements ". Enfin, les décorateurs qui assemblent les fragments de maquettes et les peignent dans l'atelier de montage et de finition. Cette répartition du travail, quasi industrielle, offre toutefois une vision idéalisée de son organisation.

Précises, détaillées, pour ne pas dire sophistiquées, les maquettes produites par l'atelier sont conçues à l'aide de matériaux divers - bois, métal, carton, papier, végétaux - pour obtenir un rendu très réaliste. De petits personnages ainsi que des éléments de contexte urbain y sont parfois apportés afin d'en accentuer le caractère illusionniste (ill. 1). Dans certains cas, la maquette est même pourvue d'un éclairage artificiel. La société a d'ailleurs déposé en 1931 un brevet d'invention intitulé "Application des sélecteurs téléphoniques aux maquettes, plans en relief, dioramas, modèles en réduction et autres objets similaires " ${ }^{19}$ dans lequel elle décrit une méthode pour éclairer à distance tout ou partie d'un objet. L'exemple développé est celui d'un modèle de salle de théâtre dont les sièges numérotés sont équipés d'une ampoule que l'on actionne grâce à un cadran téléphonique relié à la maquette par un système électrique. Le préposé à la location peut ainsi, par composition du numéro de la place, désigner un emplacement à son client. L'inventeur prend soin de préciser qu'on pourra "appliquer le même dispositif à d'autres maquettes ou objets similaires ». Certaines productions de l'atelier, dont les photographies accompagnent le reportage de Jean Léon $^{20}$, en sont d'ailleurs l'illustration, comme les vues diurne et nocturne d'une maquette de la cité-jardin de la Butte Rouge à ChâtenayMalabry ${ }^{21}$ présentées l'une au-dessus de l'autre afin d'en mesurer la métamorphose.

16. Ibid., 29 juillet 1931 , p. 3722.

17. Jean Léon, op. cit., "Les maquettes ", p. 114-116.

18. Voir Richard Klein, "Maquette : l'architecture réduite à l'art ", Quand l'architecture moderne s'exposait, 1922-1932, Nancy, Fage, 2010, p. 123-131.

19. Brevet d'invention $\mathrm{n}^{\circ} 712.742$ déposé le 6 mars 1931 par la société anonyme Les Maquettes Perfecta, Archives INPI.

20. Jean Léon, op. cit., "Les maquettes ", $\mathrm{n}^{\circ} 2$, mars 1932, p. 114.

21. Cité-jardin conçue par les architectes Joseph Bassompierre-Sewrin, Paul de Rutté et Paul Sirvin entre 1931 et 1940. 


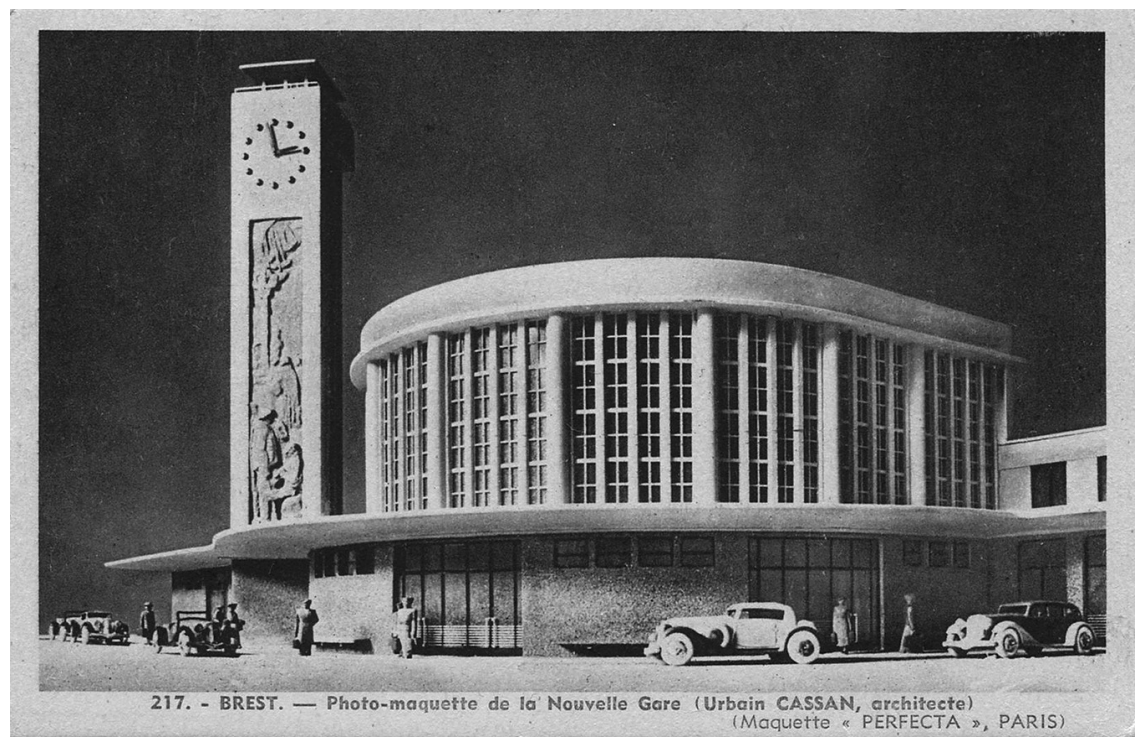

Ill. 1 : Photo-maquette de la gare de Brest (Urbain Cassan, architecte), carte postale, édition Gaby pour Artaud, n.d. (C) Collection David Liaudet.

Nouvel objet de séduction et de communication, la maquette est ici le reflet des innovations en matière d'architecture lumineuse qui se développe dans la réalité urbaine de l'entre-deux-guerres ${ }^{22}$.

Parallèlement, l'entreprise développe une véritable stratégie publicitaire. Dès 1930, elle publie dans l'almanach du commerce une annonce qui rivalise de superlatifs pour vanter l'excellence de ses productions : "Incassables, légères, $50 \%$ au-dessous des prix courants, garanties 10 ans ${ }^{23}$. L'année suivante, la société joint trois illustrations pour présenter ses réalisations et leur diversité, pratique commerciale qu'elle est seule à développer parmi ses rares concurrents. Elle choisit de reproduire une image de la maquette de l'hôtel de $\mathrm{M}^{\text {me }}$ Reifenberg de Robert Mallet-Stevens accompagnée de celles d'un turbo-alternateur pour les établissements Cail et d'un modèle de wagon frigorifique pour S.T.E.F., jeune société spécialisée dans le transport et la logistique du froid. Maquettes d'architecture et maquettes industrielles, Perfecta entend répondre à toutes les commandes. Jean Léon énumère d'ailleurs dans son article la gamme des maquettes produites par l'atelier : "plans et cartes en relief de cités-jardins, de ports, de villes entières ; réductions de machines les plus compliquées, locomotives, turbines, chaudières, navires ; reproductions complètes d'usines

22. À ce sujet, Ruth Hommelen, "Images construites. L'architecture lumineuse vue par les architectes, les éclairagistes et les photographes (1926-1939)", Éric Monin et Nathalie Simonnot (dir.), L'Architecture lumineuse, Gand, éditions Snoeck, 2012, p. 77-84.

23. Annuaire du commerce Didot-Bottin, 1930, catégorie "maquettes en relief». 


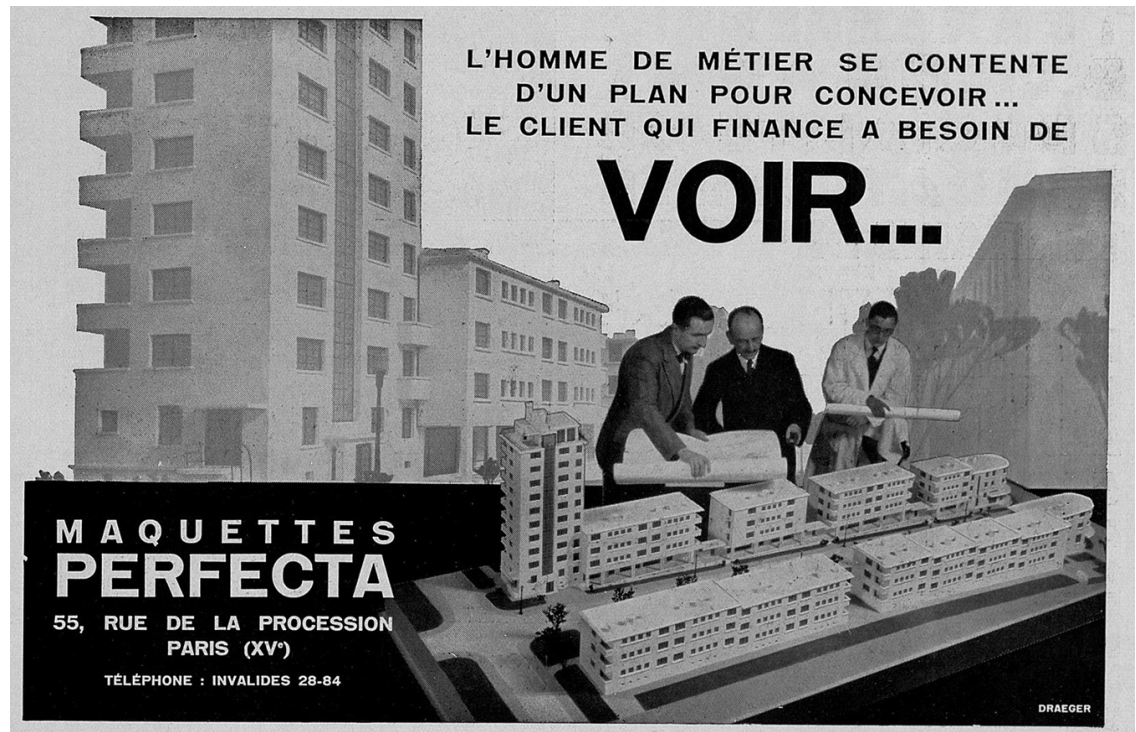

Ill. 2 : Maquettes Perfecta, publicité parue dans L'Architecture d'Aujourd'hui, no 8, novembre 1932. (c) L'Architecture d'Aujourd'hui.

entières avec tout leur outillage; maquettes d'architecture reproduisant soit des ensembles complets, soit des détails que l'architecte a voulu étudier de plus près » ${ }^{24}$.

Très active, la communication de la société devient de plus en plus incisive. À cet égard, l'impulsion d'André Bloc, fondateur de L'Architecture d'Aujourd'hui et nouvel associé de l'atelier ${ }^{25}$, est certainement décisive. Ainsi, la revue publie abondamment des photographies des modèles Perfecta, seul maquettiste qui bénéficie de la mention quasi-systématique de son crédit. L'entreprise fait également appel à des publicitaires pour concevoir une réclame qu'elle diffuse largement à partir de 1932 au sein des pages promotionnelles de journaux spécialisés. L'accroche consiste à vanter les vertus de la maquette qui permet à tous, et non seulement aux spécialistes, d'appréhender un projet architectural : "L'homme de métier se contente d'un plan pour concevoir... le client qui finance, a besoin de VOIR... ». L'image utilisée à l'appui du slogan est tout aussi claire : au premier plan trône l'imposante maquette de la cité-jardin de Châtenay-Malabry, support de dialogue et d'échanges entre l'architecte et le commanditaire tandis que le maquettiste se tient au second plan (ill. 2). À partir de 1934, l'entreprise élabore une deuxième série de publicités, conçues cette fois-ci pour occuper une pleine page. La réclame indique que les maquettes Perfecta "permettent à l'architecte la mise au point définitive de son

24. Jean Léon, op. cit., "Les maquettes ", $\mathrm{n}^{\circ} 2$, mars 1932, p. 115.

25. Nous ne connaissons pas la date exacte de l'entrée d'André Bloc dans la société. Nous savons toutefois qu'elle est intervenue après 1931. Sa démission intervient le 18 septembre 1940 (Arch. de Paris, D33U3/1178). 


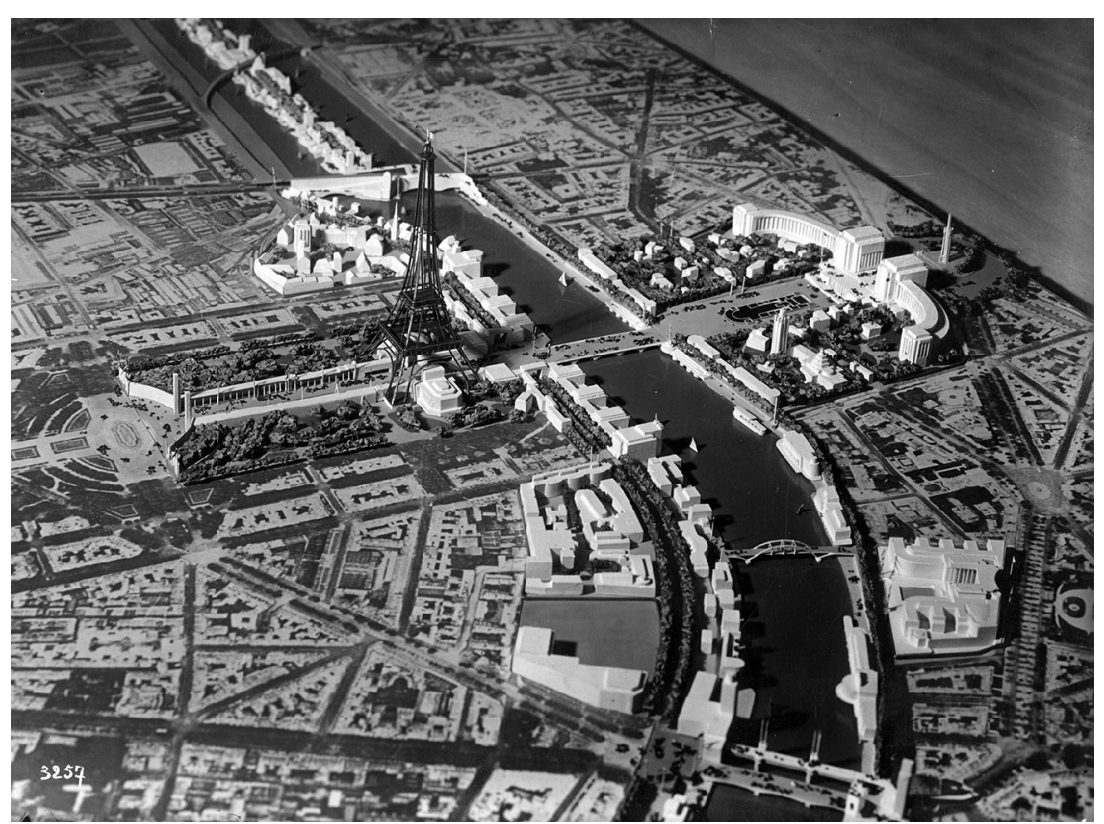

Ill. 3 : Maquette d'ensemble de l'Exposition internationale des arts et techniques appliqués à la vie moderne, Maquette Perfecta, n.d., photographie, (c) coll. privée.

étude » et « au client, la compréhension parfaite du projet... et son adoption en toute connaissance de cause ! ${ }^{26}$. Cette stratégie de communication s'avère rapidement efficace. Les commandes affluent auprès de l'atelier qui réalise de nombreuses maquettes dont nous connaissons l'existence grâce à leur publication au sein des revues ${ }^{27}$. Objet de médiation, la maquette est préconisée comme moyen de persuasion, tant pour les architectes que pour les maîtres d'ouvrage. C'est le cas pour le nouveau Trocadéro de Jacques Carlu, Louis-Hippolyte Boileau et Léon Azéma, dont le projet suscite de vives polémiques relayées par les quotidiens et les hebdomadaires ${ }^{28}$. Aux attaques de la presse, la " commission de propagande » et les architectes de l'Exposition répliquent à l'aide de conférences, d'articles, de brochures richement illustrées et de maquettes dont les images sont abondamment publiées dans la presse généraliste comme dans les revues spécialisées ${ }^{29}$ (ill. 3). Réalisées par

26. Richard Klein, "L'Architecture en réduction : art ou communication ? ", La maquette, un outil au service du projet architectural, Actes du colloque qui s'est tenu les 20-21 mai 2011 à la Cité de l'architecture \& du patrimoine, Paris, éditions des Cendres, Cité de l'architecture et du patrimoine, 2015, p. 113-122.

27. Outre L'Architecture d'Aujourd'hui, des images des maquettes Perfecta sont publiées dans les revues L'Architecture, Travaux, Le Journal des travaux publics, L'Art vivant (liste non exhaustive).

28. Se référer à Isabelle Gournay, Le nouveau Trocadéro, Liège, Mardaga, Paris, Institut français d'architecture, 1985 , p. 37-43.

29. Le fonds d'archives de Jacques Carlu conservé par le Centre d'archives d'architecture du XXe siècle contient plusieurs coupures de presse sur lesquelles figurent des photographies des maquettes. 


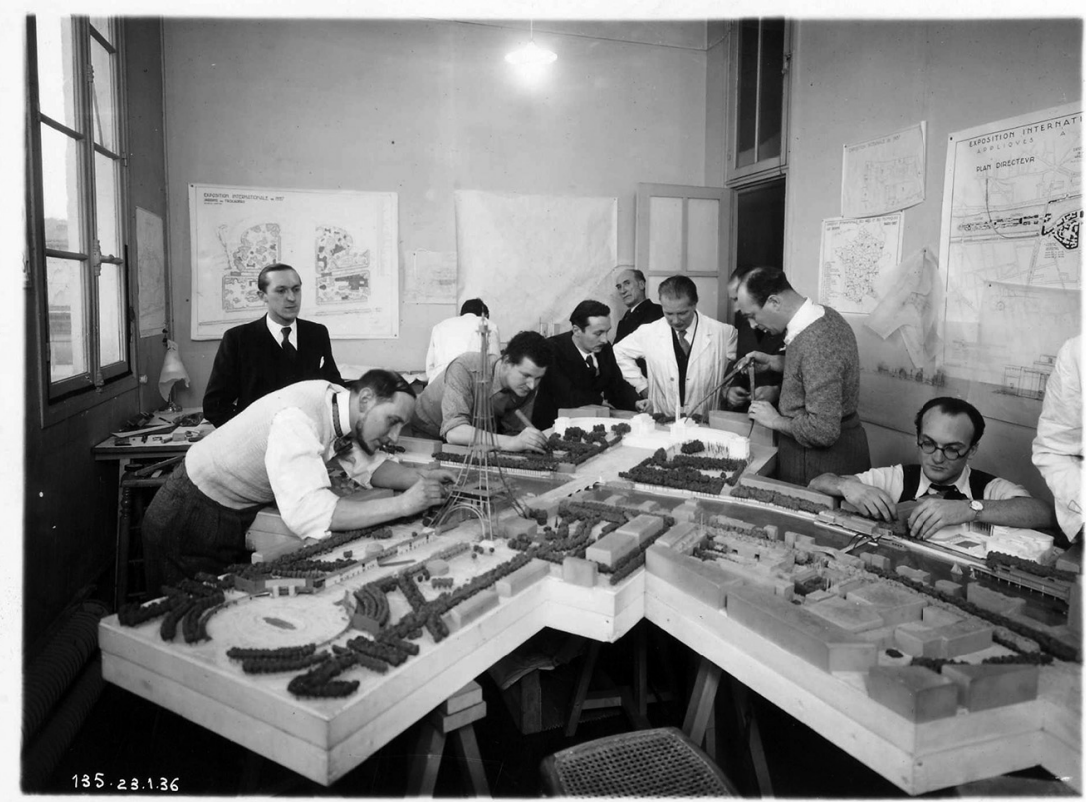

Ill. 4 : Les maquettistes de l'atelier Perfecta au travail sur la maquette de l'Exposition internationale de 1937, 23 janvier 1936, photographie. (C) Archives nationales, cliché H. Baranger.

Perfecta, la maquette du palais ${ }^{30}$ ainsi que celle de l'emprise urbaine de l'ensemble de la manifestation (ill. 4) ont certainement été obtenues grâce aux relations de Jean Léon ${ }^{31}$, ainsi qu'aux réalisations désormais nombreuses de l'atelier dont les références figurent sur les papiers à en-tête. Ces modèles sont utilisés pour présenter le projet aux décideurs ${ }^{32}$ (ill. 5), mais aussi pour convaincre l'opinion publique grâce à la diffusion de clichés dont certains sont réalisés au ras du sol afin de simuler une vision réelle et séductrice de l'architecture.

Outre la clientèle que constituent les ministères et les entreprises privées ${ }^{33}$, l'atelier réalise des modèles pour «les plus importants cabinets d'architectes à Paris

30. Cette maquette est aujourd'hui déposée dans les collections de la Cité de l'architecture et du patrimoine ( $\mathrm{n}^{\circ}$ inv. D.2013.1.1).

31. Un feuillet manuscrit concernant la société Perfecta est conservé dans le dossier «maquettistes » de l'Exposition internationale de 1937 sur lequel est laconiquement indiqué : "Perfecta maquettes, 55 rue de la Procession, Pissaro (Claude), Léon (Jean), neveu de Paul Léon " (Arch. nat., F/12/12712).

32. Une photographie de la maquette du palais a été réalisée par Baranger dans les locaux de la société en présence des architectes et du ministre du commerce. Une mention manuscrite précise l'identité des spectateurs : Jacques Carlu, Louis-Hippolyte Boileau et Georges Bonnet, ministre du commerce. Un quatrième personnage est non identifié car son image est floue (Archives d'architecture du $\mathrm{XX}^{\mathrm{e}}$ siècle, fonds Carlu, 10 Ifa 302-12).

33. Parmi les ministères, figurent celui des Travaux publics, des colonies, des postes et télégrammes ainsi que la Ville de Paris. La liste des entreprises et sociétés est également conséquente : la Compagnie 


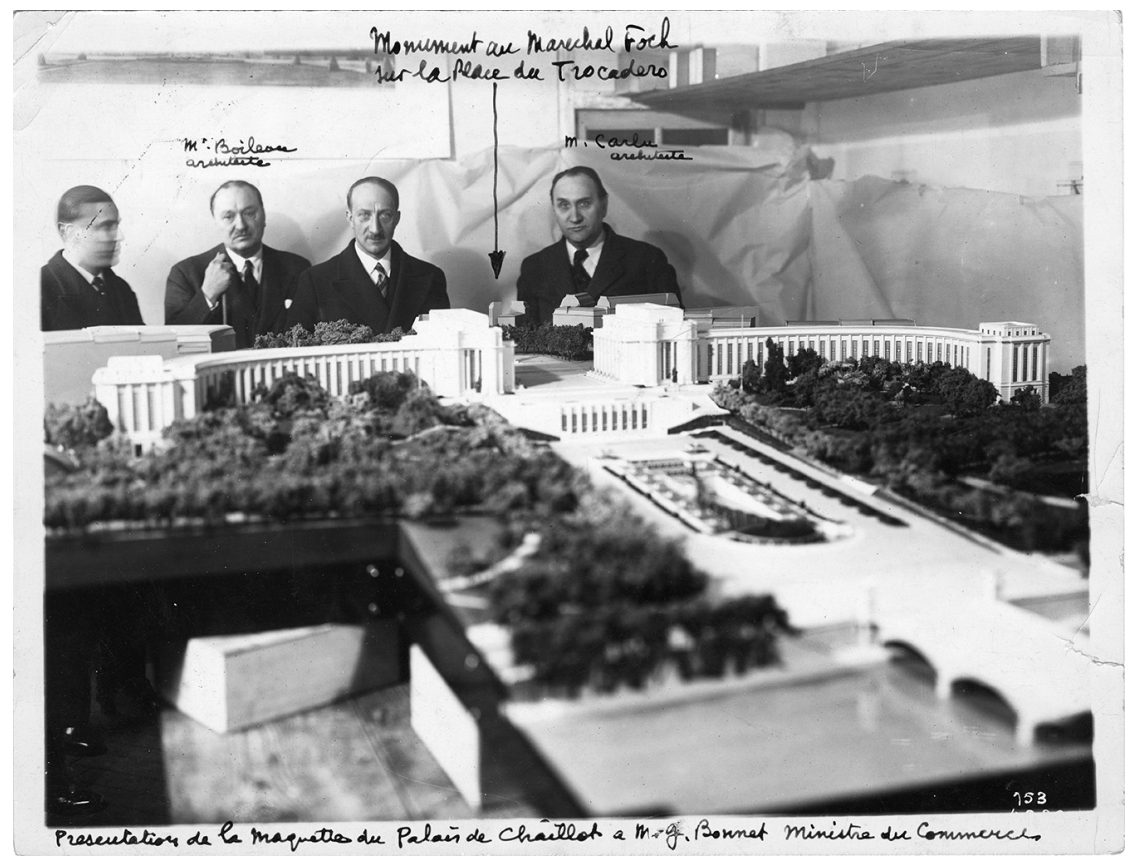

Ill. 5 : Présentation de la maquette du palais de Chaillot par les architectes Boileau et Carlu au ministre du commerce, G. Bonnet, n.d., photographie. (C) SIAF / Cité de l'architecture et du patrimoine / Centre d'archives d'architecture du XX $X^{\mathrm{e}}$ siècle, fonds Carlu, cliché H. Baranger.

et en province ${ }^{34}$ représentant des programmes de nature diverse, en particulier des ouvrages publics : l'hôpital Beaujon ${ }^{35}$, le sanatorium Martel de Janville ${ }^{36}$, le groupe scolaire de Puteaux ${ }^{37}$ ou la gare de Brest $^{38}$ en constituent quelques exemples. Le rôle des maquettes, devenues outils de communication, se double de la fonction d'illustration d'une politique publique. Son utilisation par les architectes comme par les maîtres d'ouvrage est jugée plus efficace au point de dominer peu à peu les autres représentations architecturales. Des programmes privés figurent également dans le catalogue Perfecta comme la maquette de la villa Savoye dont une photographie illustre l'article de Jean Léon paru en 1932.

générale transatlantique, les Grands réseaux de chemins de fer français, les sociétés Nord-Lumière et Ouest-Lumière, l'entreprise Boussiron, l'École spéciale des travaux publics, etc.

34. Formule qui figure sur les papiers à en-tête de la société.

35. L'hôpital Beaujon à Clichy (1933-1935) est conçu par les architectes Urbain Cassan, Louis Plousey et Jean Walter.

36. Le sanatorium Martel de Janville à Passy (Haute-Savoie) a été réalisé par Pol Abraham et Henry Jacques Le Même (1932-1937).

37. Les frères Niermans sont les architectes du groupe scolaire de Puteaux (1933-1938).

38. La nouvelle gare de Brest a été conçue par Urbain Cassan (1936-1937). 
L'atelier bénéficie également de commandes passées en vue des grandes expositions, évènements capitaux en termes d'innovation architecturale, dont les maquettes permettent de manifester la modernité. C'est le cas de René Coulon pour le pavillon de Saint-Gobain à l'Exposition internationale de 1937 tout comme pour Robert Mallet-Stevens qui conçoit le pavillon de la régie des tabacs, celui de l'électricité ainsi que celui de l'hygiène.

Enfin, plusieurs institutions muséales passent commandes auprès de Perfecta, notamment le musée des Monuments français ${ }^{39}$ ainsi que celui des Travaux publics dont les collections sont constituées de toutes pièces en vue de son ouverture en 1939. L'atelier réalise plusieurs modèles, objets d'une troisième campagne publicitaire qui vante les "très nombreuses maquettes exposées au musée permanent des travaux publics " ainsi que les stands qui les mettent en valeur. Jean Léon a en effet fondé en 1935 une société à responsabilité limitée dénommée "Stands et pavillons d'Exposition Perfecta ${ }^{40}$, afin d'élargir un peu plus l'emprise de la société sur le marché de la maquette et de son exposition.

Ainsi, à l'orée de la Seconde Guerre mondiale, la maquette n'est plus seulement " une manifestation des dimensions sculpturales ou simplement artistiques de l'architecture mais un outil professionnel fait pour convaincre les décideurs à partir d'une illusion réduite de la réalité ${ }^{41}$. Ce nouvel usage a largement bénéficié à la société Perfecta qui a élaboré une politique de communication inégalée jusqu'alors au point de devenir, en une décennie, l'entreprise emblématique de cette transformation qui a fait de la maquette un outil courant et incontournable de la pratique architecturale. L'atelier connaîtra d'ailleurs une étonnante longévité qu'aucun de ses concurrents, notamment les maquettes EXACT ou EPOC, n'a connue. Il survivra à la guerre et poursuivra son activité jusqu’à la fin des années quatre-vingt-dix.

Elvira FÉRAULT

Attachée de conservation

Cité de l'architecture et du patrimoine

39. Trois maquettes commandées au cours des années trente figurent dans les collections du musée: abbaye de Fontenay ( ${ }^{\circ}$ inv. D.MAP/CRMH 48), église abbatiale Saint-Pierre-Saint-Paul, Cluny III ( $\mathrm{n}^{\circ}$ inv. D.MAP/CRMH 49) et baptistère Saint-Jean de Poitiers ( ${ }^{\circ}$ inv. MAQ.00008).

40. Il s'agit d'une société à responsabilité limitée au capital de 25000 francs dont le siège social se trouve 6 avenue Allendy, soit à la même adresse que les Maquettes Perfecta (Archives commerciales de la France, journal officiel d'annonces judicaires et légales, 30 octobre 1935, p. 4368-4372). L'ingénieur Jean-Jacques Coulon, qui deviendra associé des Maquettes Perfecta le 18 septembre 1940 (Arch. de Paris, D33U3/1178), est l'associé de Jean Léon au sein de la société «Stands et pavillons d'Exposition Perfecta".

41. Richard Klein, op. cit., "L'architecture en réduction : art ou communication ? », p. 119. 